\title{
Temporal and spatial variations of the quiet upper chromosphere from SOHO/SUMER observations of hydrogen Lyman lines
}

\author{
R. Kariyappa ${ }^{1}$, B. A. Varghese ${ }^{1}$, and W. Curdt $^{2}$ \\ 1 Indian Institute of Astrophysics, Bangalore 560 034, India \\ 2 Max-Planck-Institut für Aeronomie, 37191 Katlenburg-Lindau, Germany
}

Received 14 December 2000 / Accepted 9 April 2001

\begin{abstract}
We have analyzed a time series of spectra in the hydrogen Lyman lines and the Lyman continuum obtained by the Solar Ultraviolet Measurements of Emitted Radiation (SUMER) spectrometer on the SOlar Heliospheric Observatory (SOHO). The time series of about 2 hours and 22 min was obtained on 1999 March 9 in a quiet region near the center of the solar disk. For our analysis, we have selected a Lyman continuum window

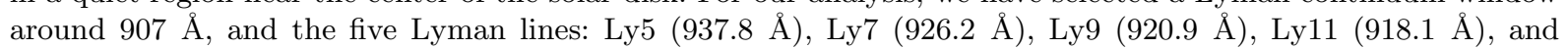
Ly15 (915.3 $\AA$ ). We derived the central intensity of these lines for a large number of locations over the network and inter-network regions. We found from their line-center intensity time series and from the power spectrum analysis that the network and inter-network regions at the upper chromosphere are associated with 5-7 min and 3 -min period of intensity oscillations, respectively. Phase difference analysis shows that there is a preponderance of upward-propagating waves in the upper chromosphere. The phase velocity was estimated to be roughly $4-5 \mathrm{~km} \mathrm{~s}^{-1}$ in the network regions. In addition, a Fast Fourier analysis has been performed in the spatial domain, all along the slit length used, for all the spectra and for the total duration of the observations. We have detected significant periodic spatial variations with Fourier transform power peaks around 9-10 arcsec and at 4 arcsec. They correspond to the scale of mesogranulation structure and the width of the supergranular boundary, respectively. This provides evidence for the existence of a meso-scale in the upper chromosphere, of the same size as observed in the photosphere and lower chromosphere by earlier studies. We notice that the size (9-10 arcsec) of the meso-scale structures appears to be the same in all Lyman lines and in the continuum, which are formed at different depths in the chromosphere.
\end{abstract}

Key words. Sun: chromosphere - Sun: oscillations

\section{Introduction}

Since its discovery by Leighton (1961), the 5-min oscillation has been the subject of a large number of observational and theoretical investigations. It has been known since the observations of Jensen \& Orrall (1963) that the solar chromosphere oscillates with a period of 180-200 s. The solar chromosphere is a highly dynamic and radiating medium. Radiation-hydrodynamical simulations of the nonmagnetic inter-network structure (cell interior) reveal remarkably good agreement between the observed and computed temporal variation of the Ca II H line (Carlsson \& Stein 1997). Selected UV lines of different species, observed with the SOHO/SUMER experiment, at the higher atmosphere exhibit a 3-min oscillatory behaviour, as in the case of CaII H and K lines (Carlsson et al. 1997; Judge et al. 1997). We have chosen five Lyman lines and a continuum. The line cores of these series are formed in the

Send offprint requests to: R. Kariyappa,

e-mail: rkari@iiap.ernet.in upper chromosphere or at the base of the transition region, above the heights so far considered in simulations. The series spans the range of heights at which strong temperature gradients can be expected and therefore can be used to diagnose the time evolution of the temperature structure. The line cores of higher Lyman lines are sensitive to the kinetic temperature (Heinzel et al. 1997) and the magnetic field. Lyman lines also play a significant role in the total energy balance of the upper chromosphere. From simulations, the time-dependent Lyman line intensities can be synthesized and compared with observations.

It is well known that there is evidence for at least three scales of motion in the solar photosphere: granulation, mesogranulation, and supergranulation. The solar surface is subject to convective and oscillatory motions, the properties of which are fundamental to understanding the physics of solar surface evolution. One of the striking properties of the observed convective motions on the Sun is the discrete spectrum of their horizontal scales. Granules have scales of order 1 arcsec, the mesogranules of order 
8 arcsec, and supergranules of order 40 arcsec. Granulation has been the subject of studies for more than a century, and its structure, physical properties, the lifetime, and evolution are rather well known both from observational work and numerical models, except at the smallest spatial scales below 0.2 arcsec, which are at present unaccessible by both ground-based and space-borne solar telescopes. Muller et al. (1992) have studied the evolution of solar mesogranules, which seem to survive for at least 3 hours. In a recent paper, Roudier et al. (1998) find the lifetimes for mesogranules to range from 16 to $185 \mathrm{~min}$. There is still disagreement as to the nature of the mesogranulation: whether it represents waves or convection; whether it is a distinct spatial regime or cannot be identified as a regime separated from granulation. Therefore, all these issues need to be better understood and related to the observed properties of the mesogranulation, particularly from an extended time sequence of simultaneous observations in many spectral lines.

The overshooting of convective motions into the solar photosphere, chromosphere, and transition region may provide a direct coupling between the atmosphere and the vigorous turbulence below the surface. Following the observation and a first attempt of interpretation of the mesogranulation by November et al. (1981), several related observations were carried out using different techniques either through intensity measurements (Koutchmy \& Lebecq 1986), statistical studies of active granules grouping (Oda 1984), or by measurements of granular flows (November et al. 1987). Recently, Hirzberger et al. (1999) have studied a time series of white-light images of 80-minute duration and have shown that the dynamics of exploding granules is strongly affected by their surroundings and that their appearance is closely related to the mesogranular flow field. Martic et al. (2000) have analyzed the solar ultraviolet filtergrams obtained during the fourth rocket flight of the Transition Region Camera (TRC) of 1985 October 25, and have derived a mesogranulation pattern with a size of $8 \mathrm{Mm}$ in the temperature minimum region. From different observations in Ca II H \& K lines (Damé \& Martic 1987, 1988; Kariyappa et al. 1994) the meso-scale phenomenon was identified in the lower chromosphere.

In this paper, we present the observations of the temporal evolution of five hydrogen Lyman lines and the continuum, simultaneously observed near the center of the solar disk in a quiet region by the SOHO/SUMER spectrometer. We discuss the periodicities seen at the locations of the network and inter-network regions observed in all the line profiles. In addition, the results on the spatial variations observed at the upper chromosphere using an extended time sequence of spectra of Lyman lines and the continuum are discussed.

\section{Observations and data reduction}

SUMER is a stigmatic, high-resolution normal-incidence spectrometer that covers the wavelength range be-

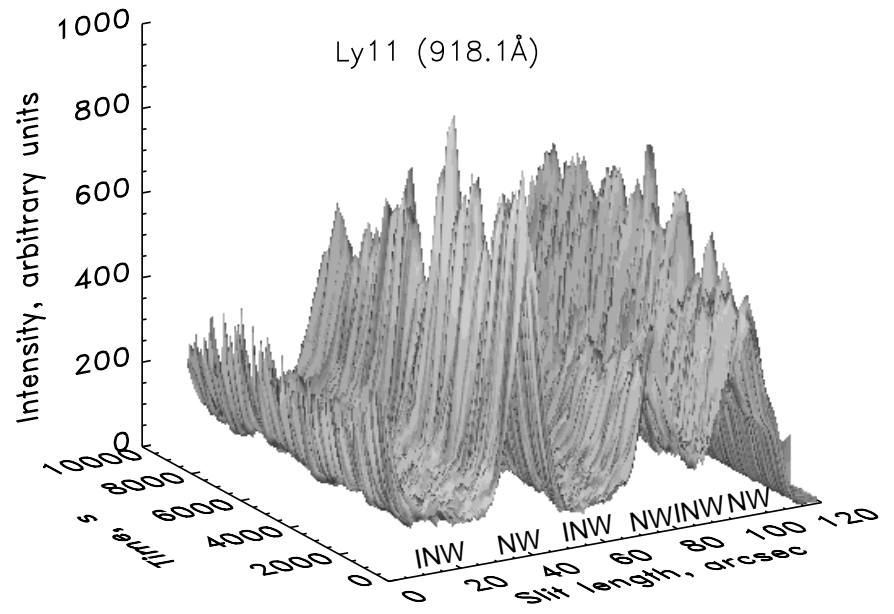

Fig. 1. Ly11 (918.1 ̊) central intensity map averaged by a smooth-3 noise filter and shown as three-dimensional plot. Here we have marked the network and inter-network regions as NW and INW respectively. We notice the intensity oscillatory nature in INW and NW.

tween 400 and $1610 \AA$. With a spatial resolution close to 1 arcsec and spectral pixels of $\approx 43 \mathrm{m \AA}$ (with subpixel resolution), it provides a unique opportunity to observe the whole hydrogen Lyman series plus the Lyman continuum. The SUMER capabilities are described in detail by Wilhelm et al. (1995), the in-flight performance is presented in Wilhelm et al. (1997) and Lemaire et al. (1997), and specific aspects related to the Lyman series spectra by Curdt \& Heinzel (1998), Heinzel \& Curdt (1999), Curdt et al. (1999).

In this paper, we have analyzed the data set observed on 1999 March 9. We used the 1 arcsec $\times 120$ arcsec slit. We have obtained a set of 306 spectra with a repetition time of about $27.72 \mathrm{~s}$. A few extra seconds were required for the solar rotation tracking, using a special, so-called half-step solar rotation compensation scheme (cf., Curdt $\&$ Heinzel 1998). The whole sequence thus lasted for $8480 \mathrm{~s}$ ( $\approx 2$ hours and $22 \mathrm{~min}$ ). The slit crossed two network cells with less brightness, a very bright network boundary lane and finally ends with a weak network lane on the southern part of the slit. In between these network regions, there are inter-network regions with an oscillatory nature. The spectra were acquired by the detector $\mathrm{A}$ on its central band of 120 spatial $\times 1024$ spectral pixels. This covered the wavelength band from 903 to $943 \AA$, including all Lyman lines from Ly5 up to the series limit, plus a portion of the Lyman continuum. This is shown in Fig. 2 of Curdt \& Heinzel (1998). We have selected five hydrogen

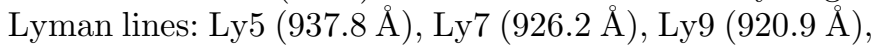
Ly11 (918.1 $\AA$ ) and Ly15 (915.3 $\AA$ ), and a Lyman continuum window around $907 \AA$ for the detailed study. For detailed line identifications see Curdt et al. (1997), Curdt \& Heinzel (1998).

The data set has been decompressed, flat-field corrected, and corrected for the geometrical distortion of the detector. We have derived the central intensity of all the line profiles for the total duration and for a large number 
Pixel location:39, Network

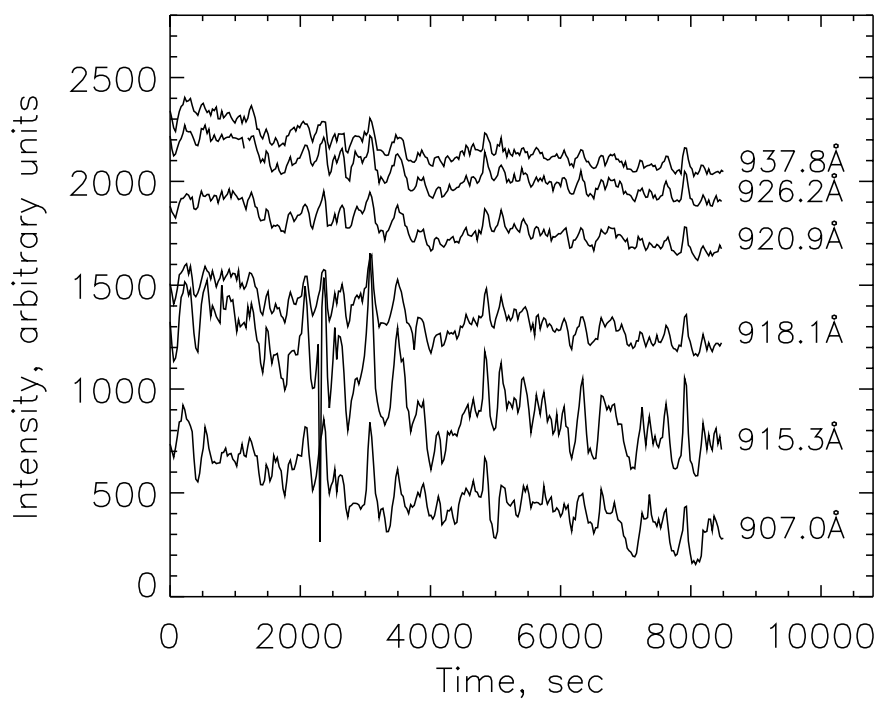

Fig. 2. Example of temporal variations in central intensity of hydrogen Lyman lines (from top to bottom): Ly5 (937.8 $\AA$ ),

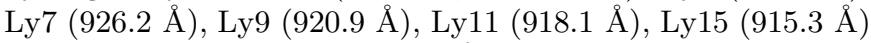
and Lyman continuum at $907.0 \AA$ using all 306 spectra of the whole data set for network (NW) region located at 39 arcsec along the slit height. For clarity the profiles have been shifted upwards along the intensity scale. Note that there is a time delay in the intensity peaks between the lines and the continuum, showing the upward propagating waves in the upper chromosphere.

of locations over the network and inter-network regions. We performed the Fast Fourier analysis in the time domain to investigate the periodicity of intensity variations. In addition, we have performed a spatial Fourier analysis all along the slit length (120 arcsec) for all the Lyman lines and the continuum, and at every time interval for the duration of our observations to determine the periodicity in the spatial scale.

\section{Results and discussions}

It is evident from the data set of 1999 March 9 that there are significant temporal and spatial variations of the intensities and shapes of all Lyman lines observed. We have derived the central intensity values for all the line profiles after calculating the center of gravity of the average profiles of these lines and applying a smooth- 3 noise filter using all 306 spectra.

\subsection{Temporal variations in bright network and inter-network regions}

In Fig. 1, we have shown a 3-D plot of the central intensity versus time and distance along the slit for the Ly11 $(918.1 \AA)$ line. It can be seen that the slit crosses three network lanes, which we have marked as NW, one of them is very bright at a pixel position around 39 and other two are less bright (at pixel positions roughly around 73
Pixel location:55 Internetwork

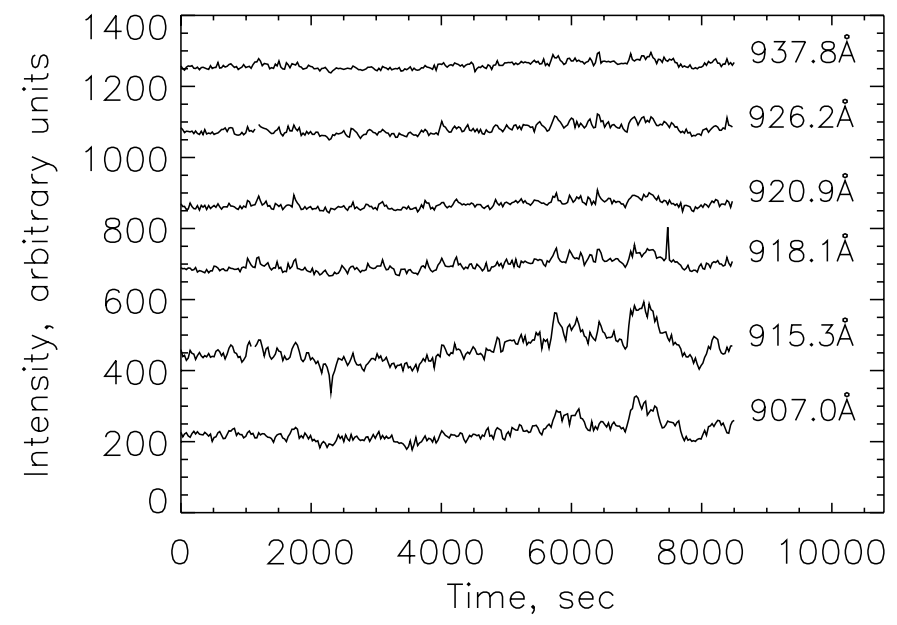

Fig. 3. Example of the temporal variations in central intensity of hydrogen Lyman lines (from top to bottom): Ly5 (937.8 $\AA$ ),

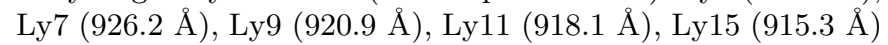
and Lyman continuum at $907.0 \AA$ using all 306 spectra of the whole data set for inter-network (INW) region located at 55 arcsec along the slit height. For clarity the profiles have been shifted upwards along the intensity scale.

and 98). In between the network lanes, we see features of the inter-network oscillatory grains (around pixel positions: $10-20,50-60$, and 80-86), which we marked as INW. We can see from the 3D-plot (Fig. 1) and the Fig. 2 time series for a typical position at 39 in the network (NW; 39) that the network regions will exhibit an oscillatory behaviour with a longer period in their intensity variations. In a similar way, we can notice from Fig. 3 for a typical position at 55 in the inter-network (INW; 55) that the inter-network regions also show an oscillatory behaviour with a shorter period and with a smaller amplitude in intensity variations. In order to determine the period of intensity oscillations, we have done the power spectrum analysis on the time series of the central intensity values for all the lines including the continuum for a number of locations over the NW and INW regions. We have suppressed the DC component in the power spectra to see clearly all the peaks. For example, in Figs. 4 and 5, we have shown the power spectrum for NW (at location 39) and INW (at location 55) regions for all the lines, respectively. We determined the level of significance for individual peaks in units of standard deviation $(\sigma)$ of the values of the power over the whole time range and show the peaks of around 5-7 min and a 3-min period with confidence levels of 96-99\% (2 $\sigma-3 \sigma)$. Although the oscillatory behaviour is clearly visible in the intensity time series plots, the power spectra do not seem to show prominent peaks. However, we identified several phase jumps with typical coherence intervals of the order of few minutes. Therefore, the FFT, as expected, did not lead to sharp peaks. We have made an attempt to break up the entire time series into shorter intervals which show more 
Pixel location:39, Network
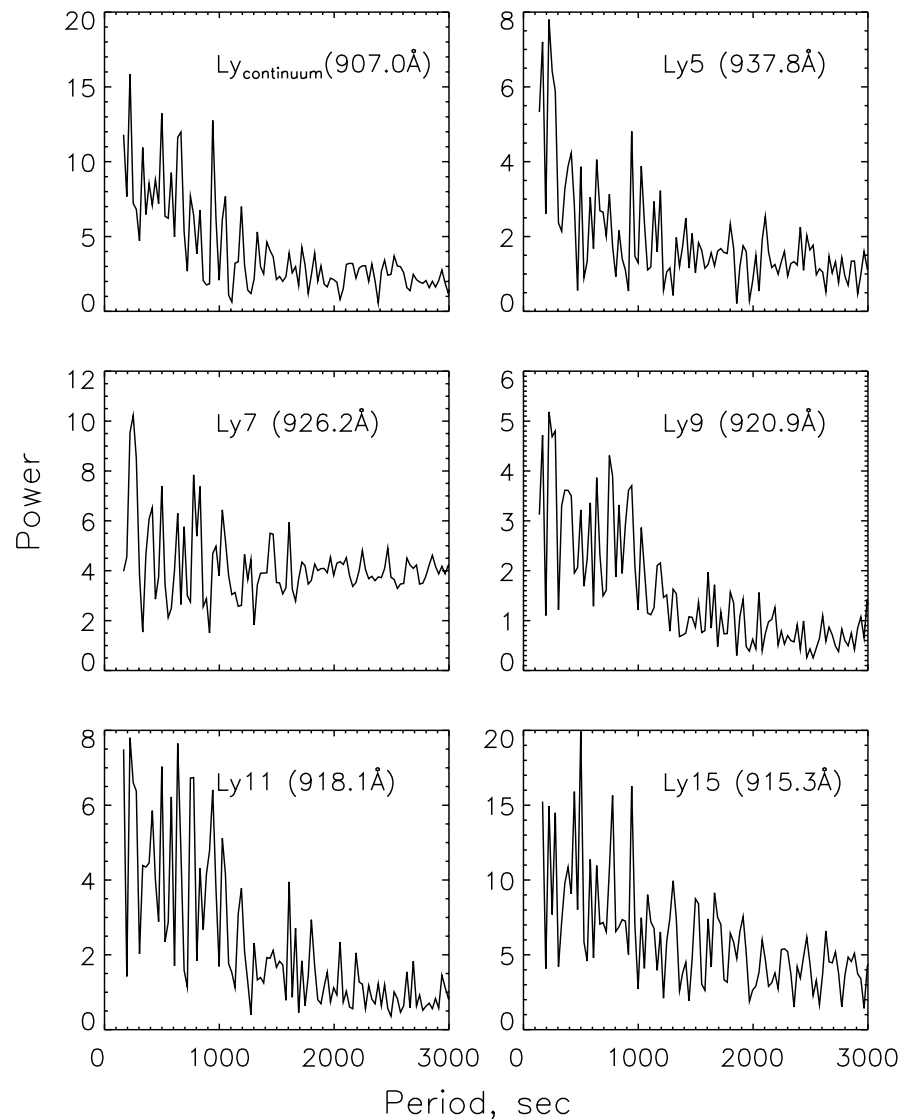

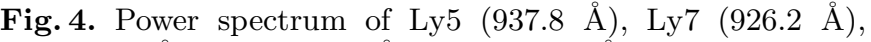

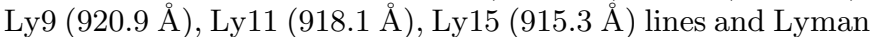
continuum at $907.0 \AA$ for the network (NW) region located at 39 arcsec along the slit height.

coherent oscillations and summed up the individual power distributions. A similar method was also suggested by Curdt et al. (1999). However, since in the Lyman line time series phase jumps frequently occur, we propose to use the wavelet analysis to confirm these results and to interpret this oscillatory behaviour as interference phenomenon of several uprising wave packets arriving from different locations in the lower chromosphere. From ground based observations in Ca II $\mathrm{H} \& \mathrm{~K}$ lines, the network and internetwork regions show 7-min and 3-min periods of intensity oscillations (e.g. Liu 1974; Cram \& Damé 1983; Lites et al. 1993; Kariyappa et al. 1994; Kariyappa 1994, 1996, 1999). It is important to note that the emission profiles of the optically thick Lyman lines span relatively large atmospheric depths (see, e.g. Fig. 1 in Vernazza et al. 1981) and, therefore, in oscillation studies one should rather use the line-center intensities rather than the integrated emission, which would allow intensity variations to mix waves from different depths.

We have determined the phase shift for the NW (39) from the prominent intensity peaks between the five observed lines and the continuum (Fig. 2). The mean time delay between the lines and continuum for the network re-
Pixel location:55, Internetwork
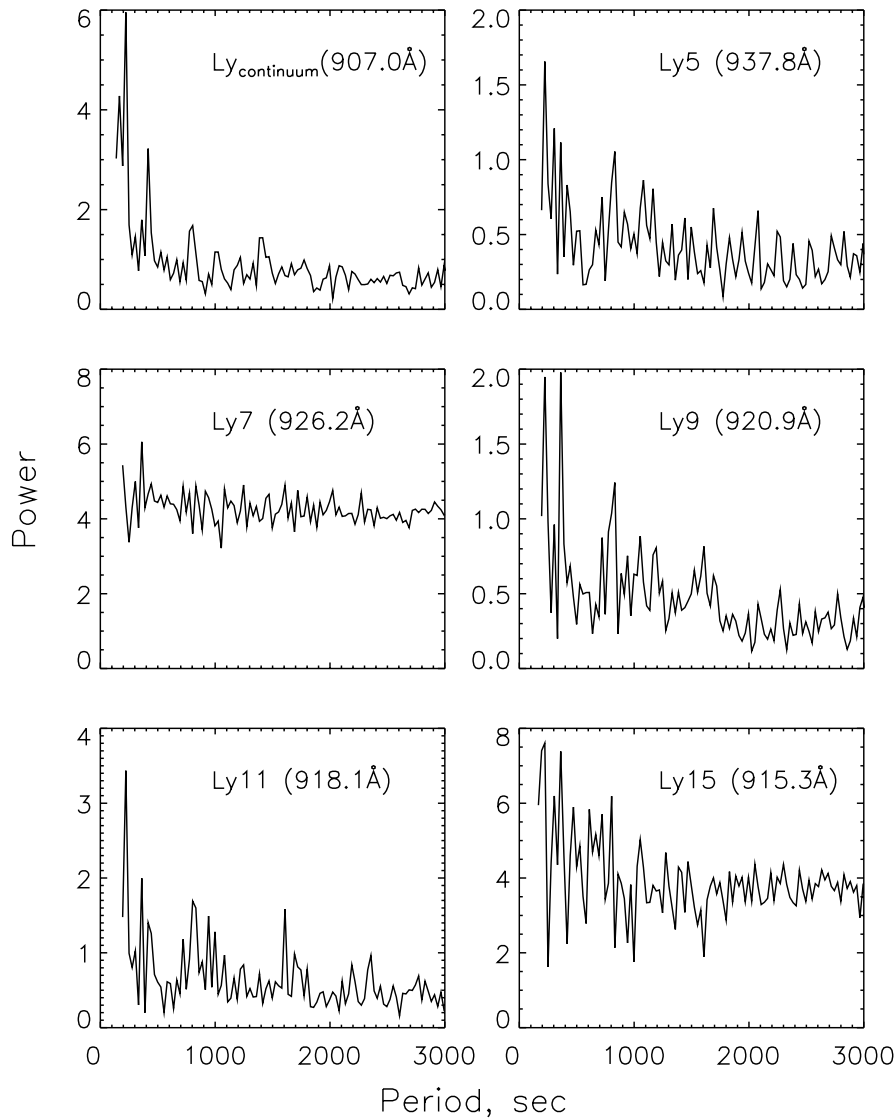

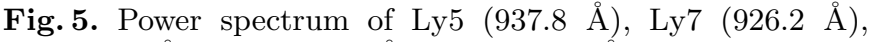

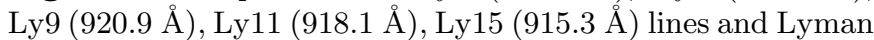
continuum at $907.0 \AA$ for the inter-network (INW) region located at 55 arcsec along the slit height.

gion, NW (39), is about $27.8 \mathrm{~s}$. This represents waves that seem to propagate upward in the solar atmosphere. The height difference between the Ly5 line and the continuum is about $150 \mathrm{~km}$ (Vernazza et al. 1981; Curdt \& Heinzel 1998). We estimated the phase velocity as roughly of 5.4 $\mathrm{km} \mathrm{s}^{-1}$. Hence, we can conclude from this analysis of long time series data of these lines that there will be a signature of upward-propagating waves in the upper chromosphere. The analysis is thus promising and can tell more about the dynamical nature and the physical properties of the waves associated with the network and inter-network regions.

\subsection{Spatial variations at the upper chromosphere}

We have derived the spatial power spectrum plots along the slit for a given time of observation. For example, in Figs. 6 and 7, we have shown the spatial power spectrum plots for all the Lyman lines and continuum for the time intervals of around $2000 \mathrm{~s}$ and $4000 \mathrm{~s}$, respectively. The spatial power spectrum plots show two significant power peaks. The first peak is around 910 arcsec, which is in consistence with the scale of mesogranulation observed in the photosphere from SOUP 
Time interval $t=2000 \mathrm{~s}$
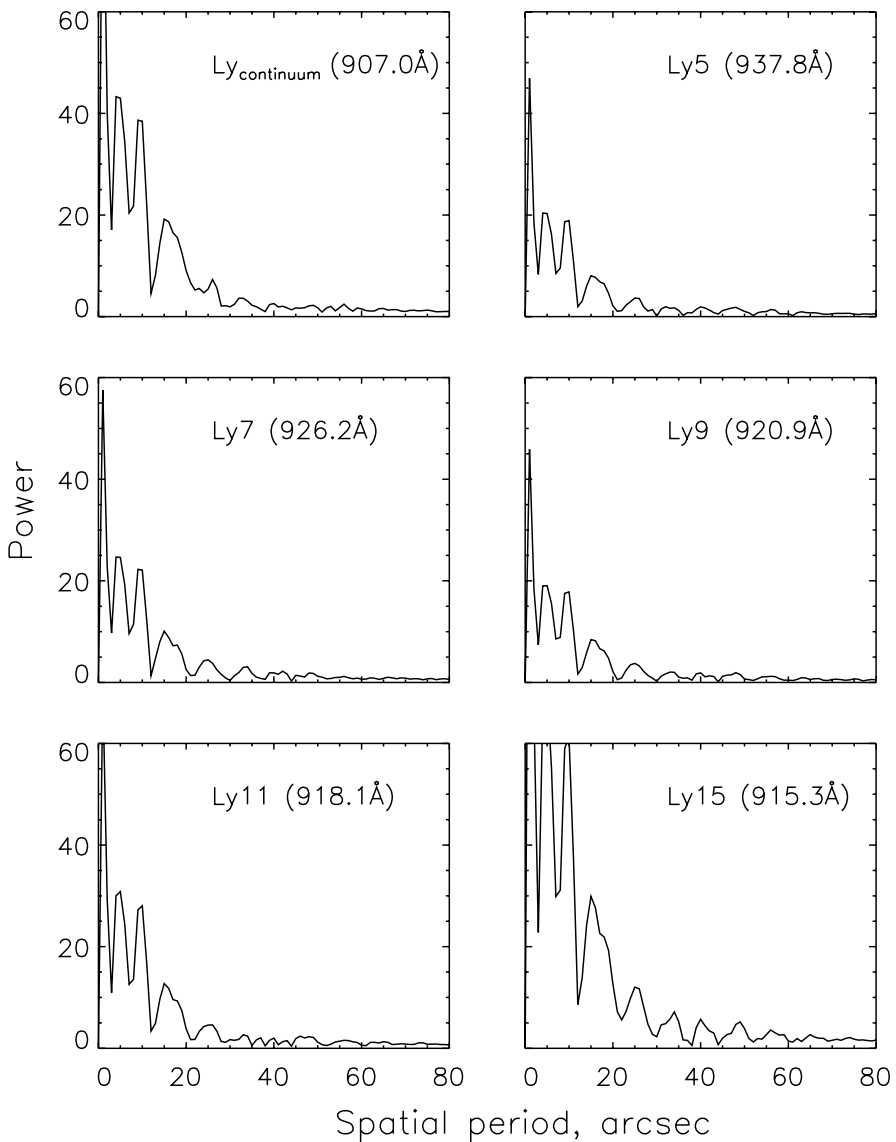

Fig. 6. Spatial power spectra of Ly continuum (907.0 $\AA$ ),

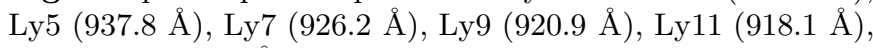
and Ly15 (915.3 $\AA$ ) for the time interval around $2000 \mathrm{~s}$ during the sequence. Note that there are two significant peaks around 9-10 arcsec (meso-scale) and at 4 arcsec (width of the supergranular boundary). Also notice that these two peaks are clearly dominant in all the lines, which are formed at different depths in the upper chromosphere.

observations (November et al. 1981), in the temperature minimum region from TRC ultraviolet filtergrams (Martic et al. 2000), as well as in the lower chromosphere from Ca II H \& K lines (Damé \& Martic 1987, 1988; Kariyappa et al. 1994), and the second peak is seen at 4 arcsec, which may be related to the width of the supergranular boundary. In addition to these two peaks, we find the smaller amplitude peaks at higher spatial periods. They may be related to the sizes of the supergranular cells. We have looked for potential instrumental effects that could create periodic signals in the spatial domain, and have analyzed the detector non-uniform response, often referred to as flatfield. The micro-channel-plate of the detector has more or less regular geometrical structures which lead to variations of the effective size of the pixels. The corresponding effect on the detector responsivity, which is taken care of by the flatfield matrix revealed a clear peak near pixel 26 , but no prominent peak at higher frequencies. This excludes that residual effects of the flatfield correction could possibly be responsible for the signals we have revealed and demon-
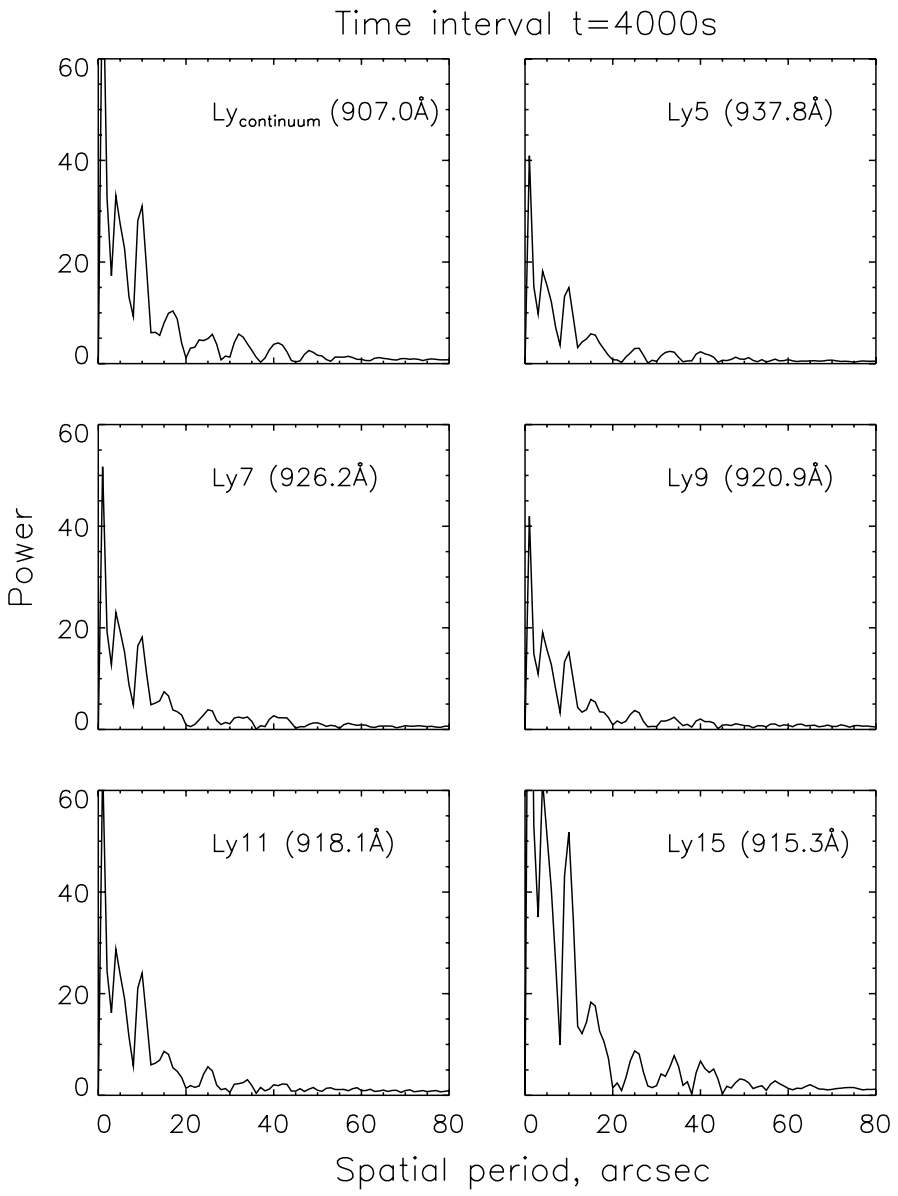

Fig. 7. Same as Fig. 6, but the spatial power spectra for the time interval around $4000 \mathrm{~s}$ during our observing sequence. Note that the scale and amplitude of the peaks are more or less the same as for $2000 \mathrm{~s}$. This suggests that mesocells will have a lifetime more than our total observing period.

strates their solar nature. Therefore, we conclude that the maximum around 9-10 arcsec corresponds to meso-scale structure. The study of the temporal variations of the Lyman emission line profiles (discussed in Sect. 3.1) reveals that the peaks in the power spectra at spatial period 9-10 arcsec are not due to the solar 3-minute oscillations, but they are due to a slowly varying phenomenon of mesoscale structure. This result from SUMER observations of higher hydrogen Lyman lines is new and suggests a mesoscale structure in the upper chromosphere. After carefully examining the power peak values, it is interesting to note that the size of the meso structures will be the same in all the Lyman lines including continuum, which are formed at different depths in the chromosphere. We can remark that further up in the atmosphere this 9-10 arcsec dimension will keep the same spatial extent, even up to the transition zone (Dere et al. 1986), where only a slight increase (11 arcsec) in dimension occurs. The meso-scale structure has been seen all along in the photosphere temperature minimum region, in the lower and upper chromosphere, 
and this result suggests that there may be an overshooting of convective motions from layers lying below.

The observed periodical brightenings on a meso-scale spatial extent above the granulation certainly gives us additional information on the processes governing the structures in that region of the solar atmosphere. The relationship between the time evolution and periodical brightenings of the meso-scale structure has to be confirmed with still a longer duration of observations. We have studied all the spatial power spectrum plots taken for all the lines for the entire duration of our observations. We find that the size of the meso structures appears to be the same in all the spectral lines and at the continuum. The amplitude of the power peak remains the same for a given line and time during our observations. However, we see a slightly larger amplitude in the power peaks in the case of Lyman continuum (at 907.0 $\AA$ ) and Ly15 (915.3 $\AA$ ) line compared to the rest of other lines, and these two are formed rather in the lower atmosphere. From this result, we can infer that the lifetime of the meso-scale structures is more than several hours. This is in good agreement with the findings reported by Muller et al. (1992). However, in order to clarify: (i) whether we could see another meso-scale structure at the previous location or not; (ii) whether it changes with time and different positions over the solar disk; and (iii) whether the meso-scale structure observed at the upper chromosphere and photosphere is spatially related, we propose to analyse and investigate long-duration simultaneous observations of the same region on the Sun made by the SUMER and MDI experiments. Since the lifetime of the mesocells seems to be more than 3 hours, our present time sequence of observation is too short to comment on the temporal evolution of the meso-scale structures.

Acknowledgements. The SUMER project is financially supported by DLR, CNES, NASA, and the ESA PRODEX Programme (Swiss Contribution). SUMER is part of $S O H O$, the SOlar and Heliospheric Observatory, of ESA and NASA. We highly appreciate Dr. Luc Damé's stimulating discussion on this research. We wish to thank Dr. Klaus Wilhelm for critical comments and suggestions, which helped to improve the manuscript. We are thankful to Dr. R. Muller who as a referee made valuable comments and suggestions.

\section{References}

Carlsson, M., \& Stein, R. F. 1997, ApJ, 481, 500

Carlsson, M., Judge, P. G., \& Wilhelm, K. 1997, ApJ, 486, L63

Cram, L. E., \& Damé, L. 1983, ApJ, 272, 355

Curdt, W., Feldman, U., Laming, J. M., et al. 1997, A\&AS, 126,281
Curdt, W., \& Heinzel, P. 1998, ApJ, 503, L95

Curdt, W., Heinzel, P., Schmidt, W., et al. 1999, EPS Conf., ed. A. Wilson, ESA-SP 448, 177

Damé, L., \& Martic, M. 1987, ApJ, 314, L15

Damé, L., \& Martic, M. 1988, Advances in Helio- and Asteroseismology, ed. J. Christensen-Dalsgaard \& S. Frandsen, IAU Symp., 123, 433

Dere, K. P., Bartoe, J.-D.F., \& Brueckner, G. E. 1986, ApJ, 305, 947

Heinzel, P., \& Curdt, W. 1999, Solar Magnetic Fields and Oscillations, ed. A. Hofmann, \& J. Staude, ASP Conf. Ser., 184, 201

Heinzel, P., Schmieder, B., \& Vial, J.-C. 1997, Proceedings of the Fifth SOHO Workshop, ESA SP-404: Noordwijk: ESA, 427

Hirzberger, J., Bonet, J. A., Vázquez, M., \& Hanslmeir, A. 1999, ApJ, 527, 405

Jensen, E., \& Orrall, F. Q. 1963, ApJ, 138, 252

Judge, P. G., Carlsson, M., \& Wilhelm, K. 1997, ApJ, 490, L195

Kariyappa, R. 1994, Solar Phys., 154, 19

Kariyappa, R. 1996, Solar Phys., 165, 211

Kariyappa, R., Sivaraman, K. R., \& Anadaram, M. N. 1994, Solar Phys., 151, 243

Kariyappa, R. 1999, High Resolution Solar Physics: Theory, Observations, and Techniques, ed. T. R. Rimmele, K. S. Balasubramaniam, \& R. R. Radick, 183, 420

Koutchmy, S., \& Lebecq, C. 1986, A\&A, 169, 323

Leighton, R. B. 1961, Aerodynamic Phenomena in Stellar Atmospheres, ed. R. N. Thomas, IAU Symp., 12, 321

Lemaire, P., Wilhelm, K., Curdt, W., et al. 1997, Solar Phys., 170, 105

Lites, B. W., Rutten, R. J., \& Kalkofen, W. 1993, ApJ, 414, 345

Liu, S. Y. 1974, ApJ, 189, 359

Martic, M., Damé, L., \& Kariyappa, R. 2000, A\&A, under revision

Muller, R., Auffret, H., Roudier, Th., et al. 1992, Nature, 356, 322

November, L. J., Toomre, J., Gebbie, K. B., \& Simon, G. W. 1981, ApJ, 245, L23

November, L. J., Simon, G. W., Tarbell, T. D., Title, A. M., \& Ferguson, S. H. 1987, Proceedings of the Theoretical Problems in High Resolution Solar Physics II, ed. A. Athay, \& D. S. Spicer, NASA Conf., Publication 2483, 121

Oda, N. 1984, Solar Phys., 93, 243

Roudier, Th., Malherbe, J. M., Vigneau, J., \& Pfeiffer, B. 1998, A\&A, 330, 1136

Vernazza, J. E., Avrett, E. H., \& Loeser, R. 1981, ApJS, 45, 635

Wilhelm, K., Curdt, W., Marsch, E., et al. 1995, Solar Phys., 162, 189

Wilhelm, K., Lemaire, P., Curdt, W., et al. 1997, Solar Phys., 170, 75 